\title{
NEUROPATÍA ÓPTICA ISQUÉMICA ANTERIOR MIGRAÑOSA
}

\section{MIGRAINEOUS ANTERIOR OPTIC ISCHEMIC NEUROPATHY}

\author{
GONZÁLEZ-MARTÍN-MORO J ${ }^{1}$, PILO-DE-LA-FUENTE B ${ }^{2}$, MORENO-MARTÍN P ${ }^{1}$
}

\section{RESUMEN}

Caso clínico: Se presenta el caso de un paciente varón de 22 años de edad que acudió refiriendo visión borrosa y cefalea de cuatro días de evolución. Fue diagnosticado inicialmente de neuritis óptica desmielinizante ante el hallazgo en la resonancia magnética de una lesión periventricular, y tratado con metilprednisolona intravenosa.

Discusión: Ante la ausencia de mejoría de la función visual tras seis meses de evolución, es probable que el diagnóstico inicial fuera erróneo y que el paciente sufriera un cuadro de neuropatía óptica isquémica anterior migrañosa. Se revisa la escasa literatura publicada en relación con este tema y el papel de la migraña como factor de riesgo cardiovascular.

Palabras clave: Neuropatía óptica isquémica anterior, neuropatía desmielinizante, migraña con aura, infarto migrañoso, factores de riesgo cerebro-vascular.

\begin{abstract}
Case report: We report the case of a 22-year-old man who presented with headache and blurred vision which had started four days previous. A periventricular lesion was found in the magnetic resonance imaging. The patient was diagnosed with demyelinating neuritis and treated with intravenous methylprednisolone.

Discusion: After six months visual function had not improved, so the initial diagnosis was probably erroneous. It is likely that the patient suffered from migraineous optic ischemic neuropathy. In this paper we review the scarce literature about this topic, and the role of migraine as a cardiovascular risk factor (Arch Soc Esp Oftalmol 2009; 84: 473-476).
\end{abstract}

Key words: Anterior ischemic optic neuropathy, demyelinating neuropathy, migraine with aura, migraineous infarction, cerebro-vascular risk factors.

\section{CASO CLÍNICO}

Se presenta el caso de un varón de 22 años de edad que acudió a urgencias refiriendo cefalea y pérdida visual de cuatro días de evolución. Como antecedentes personales destacar que el paciente presentaba una historia familiar y personal clara de migraña con aura, practicaba deporte de forma regular y llevaba una dieta especial de gimnasio que incluía suplementos de carbohidratos y proteínas.

\footnotetext{
Recibido: 7/4/08. Aceptado: 28/9/09.

Hospital de la Princesa. Madrid. España.

${ }^{1}$ Licenciado en Medicina. Servicio de Oftalmología.

${ }^{2}$ Licenciado en Medicina. Servicio de Neurología. Hospital General Universitario Gregorio Marañón. Madrid. España.

Trabajo presentado en el 17 Congreso de la Sociedad Internacional de Neuro-Oftalmología (INOS) (San Francisco, 2008).

Correspondencia:

Julio González Martín-Moro

Servicio de Oftalmología

Hospital de la Princesa

C/. Diego de León, 62

28006 Madrid

España

E-mail: juliogmm@yahoo.es
} 


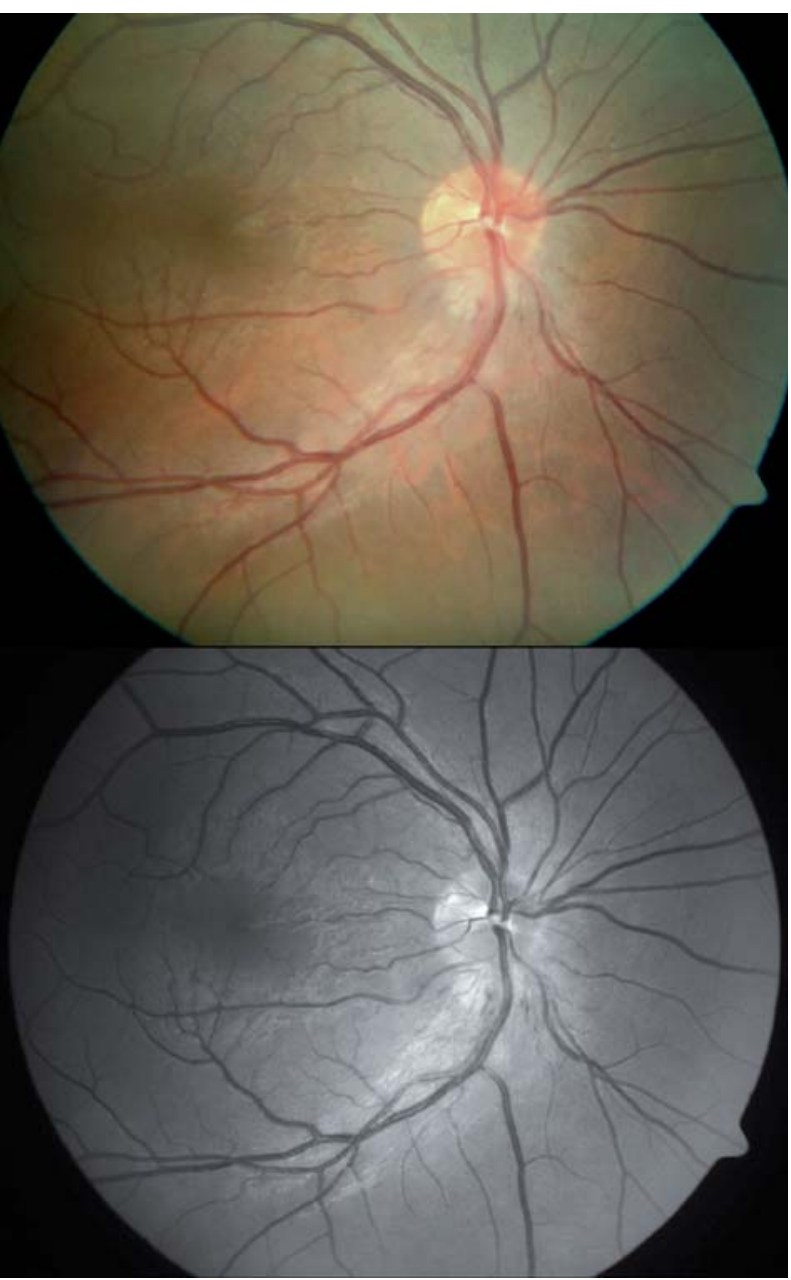

Fig. 1: Retinografía del OD en el momento del diagnóstico. Se aprecia intenso edema de papila circunscrito al polo inferior de la misma (imagen superior). Con luz aneritra el edema de la capa de fibras nerviosas se hace más evidente (imagen inferior).

Su agudeza visual (AV) era 20/20 en ambos ojos. La visión del color era normal (láminas de Ishihara 9/9). Presentaba un defecto pupilar aferente relativo leve $(1+/ 4+)$ en su ojo derecho. En la exploración de fondo de ojo se evidenciaba la presencia de edema localizado en el polo inferior de la papila, y la capa de fibras nerviosas correspondiente en el ojo derecho (OD) que se cuantificó con tomografía de coherencia óptica (OCT). En el ojo izquierdo (OI) la papila presentaba unos bordes nítidos y una mínima excavación. La perimetría computerizada demostró la existencia de un defecto arciforme superior profundo con muy buena correlación con la exploración papilar. Todos los parámetros de

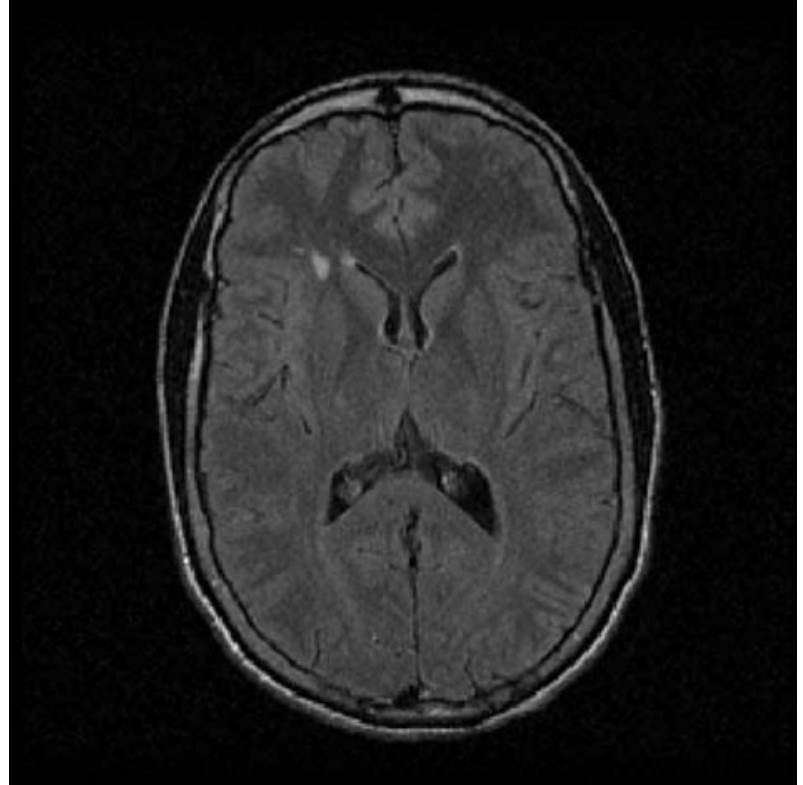

Fig. 2: En la resonancia magnética aparece una lesión, que dada la edad del paciente, se etiquetó en principio como probablemente desmielinizante.

laboratorio fueron normales, excepto un bajo nivel de colesterol HDL, y en consecuencia un alto ratio de colesterol total/colesterol HDL (perfil lipídico: VLDL 20 mg/dl, LDL 118 mg/dl, HDL 10 md/dl,
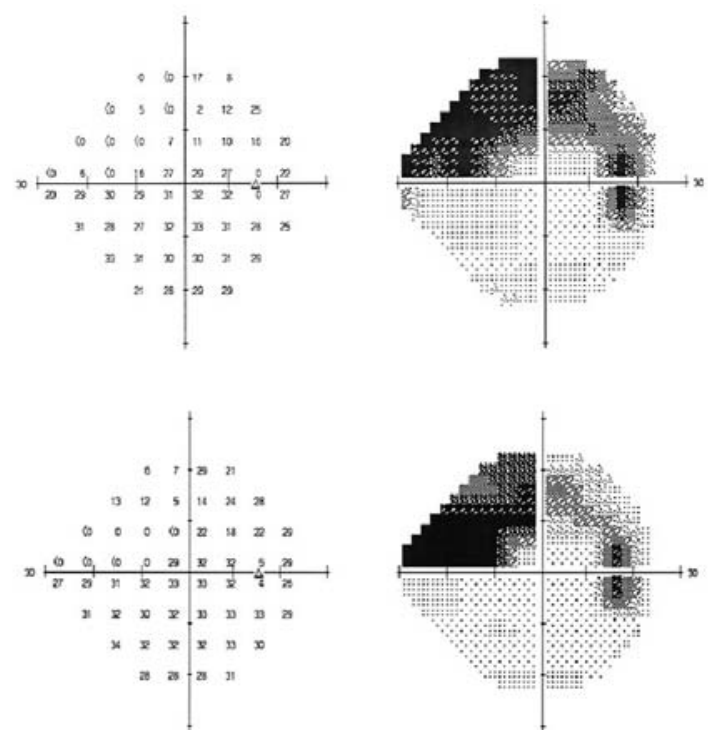

Fig. 3: Defecto arciforme superior en el OD, en el momento del diagnóstico y al cabo de 3 meses, que muestra una correlación extraordinaria con la exploración papilar y con la tomografía de coherencia óptica. 

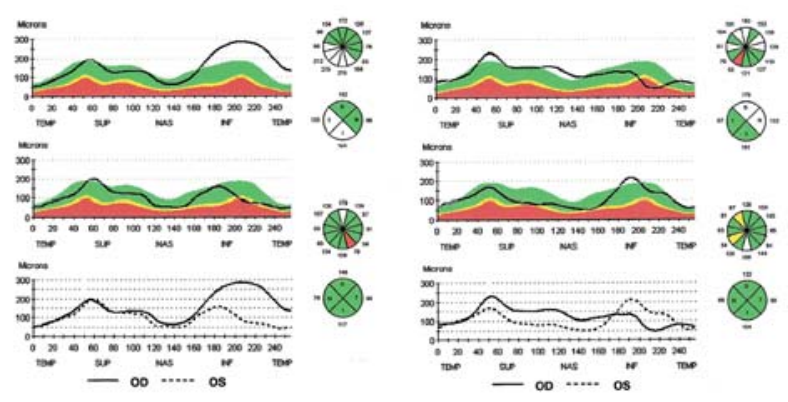

Fig. 4: Tomografía de coherencia óptica de la capa de fibras nerviosas del $O D$, en el momento del diagnóstico y al cabo de 3 meses. El edema inicial ha dado paso a la atrofia y se ha producido un adelgazamiento muy significativo en la zona afecta.

colesterol total/colesterol HDL 14,8). El resto de la exploración neurológica era normal.

El paciente fue ingresado a cargo del servicio de Neurología y sometido a un estudio completo que incluyó punción lumbar, siendo todos los parámetros normales. Se realizó Tomografía computerizada (TC) craneal y resonancia magnética (RM) cerebral, que pusieron de manifiesto la presencia de una lesión periventricular inespecífica de localización frontal. Dada la edad del paciente el cuadro se etiquetó como probablemente desmielinizante y se inició tratamiento con metilprednisolona intravenosa (1 gramo/día) durante 3 días.

En la exploración posterior el paciente ha permanecido estable, sin que se objetiven cambios significativos en su campo visual ni en su agudeza visual. Al cabo de tres meses se había producido una resolución total del edema de papila, con una pérdida de espesor localizada en la capa de fibras nerviosas del área correspondiente. Esta evolución es la típica de una neuropatía óptica isquémica anterior, por lo que con posterioridad se ha ampliado el estudio de trombofilia (homocisteína, antitrombina III funcional, protrombina 20210, anticoagulante lúpico, factor VII, Factor V Leiden, proteina C y proteína $\mathrm{S}$ ), siendo todos los resultados negativos. La lesión frontal no ha modificado su aspecto en estudios posteriores de neuroimagen.

\section{DISCUSIÓN}

Presentamos el caso de un paciente que acudió a urgencias por pérdida visual en el contexto de un episodio de migraña con aura, y fue diagnosticado inicialmente de neuritis óptica desmielinizante. La evolución natural de estos procesos es la recuperación espontánea de la mayor parte de la función visual. Esta recuperación es más rápida si el paciente es sometido a tratamiento con corticoides intravenosos. Sin embargo la relación cronológica con el episodio migrañoso, la ausencia de dolor con los movimientos oculares en el momento del diagnóstico y sobre todo la evolución posterior del paciente hace mucho más probable que el paciente sufriera un cuadro de naturaleza isquémica (1).

La neuropatía óptica isquémica anterior no arterítica (NOIANA) es una entidad que aparece sobre todo en pacientes de edad avanzada, constituyendo la morfología de la papila el mayor factor de riesgo y contribuyendo los factores de riesgo cardiovascular clásicos con un peso que ha sido discutido en distintos estudios. Trabajos recientes demuestran que la neuropatía óptica isquémica no es tan rara en pacientes por debajo de los 50 años de edad (2). Sin embargo, la mayor parte de los casos descritos en pacientes tan jóvenes se producen de forma perioperatoria en relación con cirugías con una importante pérdida de volumen sanguíneo (sobre todo cirugía espinal) (3).

La migraña aumenta el riesgo de infarto en la población general sólo muy ligeramente, pues el peso de los restantes factores de riesgo cerebrovascular es mucho mayor. Este bajo peso específico, explica que a pesar de ser la migraña una enfermedad tan prevalente sean tan pocos los casos publicados de eventos isquémicos de causa migrañosa (4). Sin embargo en la población joven, excluidos los factores de riesgo convencionales, el peso relativo de la migraña se incrementa de forma significativa, y algunos estudios estiman un aumento del riesgo del orden de 3 a 5 veces. Aún así en casi todos los casos publicados aparecen otros factores de riesgo asociados (5). Habitualmente estos infartos afectan a la circulación posterior (arteria cerebral posterior), sin embargo también hay descritos eventos isquémicos por afectación de la circulación retiniana y algunos casos de neuropatía óptica isquémica como el que referimos. La mayor parte de los casos de neuropatía isquémica descritos, se refieren a formas anteriores, pero en la literatura también aparecen recogidos dos casos de neuropatía óptica isquémica posterior de causa migrañosa (1).

Creemos que en nuestro paciente la anatomía del disco óptico y los altos valores de colesterol han podido comportarse como factores de riesgo, con- 
virtiéndose el episodio migrañoso en el desencadenante final del evento isquémico. Aunque la dieta de gimnasio que seguía no contenía andrógenos, considerando que la composición de los suplementos dietéticos no era muy clara, y que pudiera existir una posible asociación causal se ha recomendado al paciente que deje de ingerirlos y se ha iniciado tratamiento hipolipemiante y antiagregante. La lesión frontal no se ha modificado en los estudios posteriores de neuroimagen, por lo que consideramos que probablemente es isquémica y guarda también relación con el cuadro migrañoso.

\section{BIBLIOGRAFÍA}

1. Lee AG, Brazis PW, Miller NR. Posterior ischemic optic neuropathy associated with migraine. Headache 1996; 36: 506-510

2. Preechawat P, Bruce BB, Newman NJ, Biousse V. Anterior ischemic optic neuropathy in patients younger than 50 years. Am J Ophthalmol 2007; 144: 953-960.

3. Vaphiades M. Perioperative posterior ischemic optic neuropathy. Surv Ophthalmol 2005; 50: 15-26.

4. O'Hara M, O'Connor PS. Migrainous optic neuropathy. J Clin Neuroophthalmol 1984; 4: 85-90.

5. Trobe JD. The neurology of vision. New York: Oxford University Press. 2001: 295. 